\title{
"Procedimiento para el diseño de escalas de medición e indicadores de control de la gestión de las competencias de procesos clave"
}

DOI: $10.46932 / \mathrm{sfjdv2n1-084}$

Received in: November 1st, 2020

Accepted in: December 30th, 2020

Eulalia M. Villa González Del Pino

$\mathrm{PhD}$. Ingeniería Industrial.

Docente Investigador. Facultad de Ingeniería Industrial.

Universidad de Guayaquil.

Ave. Dr. Raúl Gómez Lince y Ave. Dr. Juan Tanca Marengo. Guayaquil Ecuador.

E-mail: eulaliamariavilla7258@gmail.com

Ramón A. Pons Murguía

PhD. Ingeniería Industrial.

Docente Investigador. Facultad de Ingeniería Industrial.

Universidad de Guayaquil.

Ave. Dr. Raúl Gómez Lince y Ave. Dr. Juan Tanca Marengo. Guayaquil Ecuador.

E-mail: rpons2015@gmail.com

Yanko Bermùdez Villa

MSc. Industrial Engeneering

Quality Consultant.

Orlando, Florida 32822, USA.

E-mail: yankobv30@gmail.com

\section{Harold Alexis Pérez Olivera}

MSc. Ingeniería Industrial

Consultor Black Belt en Lean Six Sigma

Corporación Universitaria Americana.

20 de Julio y Calle 72. No. 41C-64. Barranquilla. Colombia.

E-mail: hperez@coruniamericana.edu.com

\section{RESUMEN}

La gestión de competencias de procesos clave de las organizaciones, como parte del Enfoque Basado en Competencias (EBC), es un camino poco explorado actualmente, aunque constituye una perspectiva que les permite desarrollar ventajas competitivas sostenibles en la creación de valor, para los clientes e implicados.

Por lo anterior, el presente trabajo se ha propuesto la aplicación de un procedimiento para mejorar el control de la gestión de las competencias de procesos clave, bajo los enfoques de Gestión por competencias y de procesos. Como parte de una investigación de mayor cobertura, en este trabajo se obtiene la validación del procedimiento en un proceso clave priorizado (Selección e integración del Recurso Humano).

En la integración de postulados universales acerca de la EBC, la gestión por procesos, y el control de gestión, radica su valor teórico; su utilidad metodológica, en el desarrollo sistémico de herramientas y 
técnicas de diversos enfoques de gestión ya referidos, que facilitan su aplicación; sus valores práctico y social se ponen de manifiesto en su contribución a la gestión integrada de las competencias en sus tres niveles: organizacional, de procesos y profesional. Su valor económico radica en el mejoramiento de la eficacia y efectividad validada durante su aplicación.

Palabras clave: Competencias básicas de procesos, gestión de competencias, mejora continua de procesos y competencias, efectividad de las mediciones.

\section{INTRODUCCIÓN:}

Hoy día, las organizaciones buscan estrategias que les permitan asumir los dinámicos y complejos cambios del entorno, referidos fundamentalmente a la evolución de los mercados y la introducción de nuevas tecnologías y métodos de trabajo en los procesos en general (Villa, 2006; Bermúdez, 2008; Pons, et. Al., 2009; Cardona, 2011). Por lo que la constante búsqueda de ventajas competitivas significa un reto del día a día para las organizaciones, con independencia del tipo de procesos que la compongan. Por ello es que, enfoques modernos de gestión tales como el de competencias (EBC), el de procesos (GxP) y el control de gestión moderno (CG), se eligen, con carácter sistémico y base científica, muchas veces de manera integrada, para lograr dichas ventajas. Para alcanzar este resultado, se requiere de la gestión de las competencias en los tres niveles: organizacional, de procesos y de puesto de trabajo (Hernández, 2008; López, 2008; Jackson, 2014; Cabrera, M. et.al; 2020). Sólo mediante la gestión de los tres niveles de competencias de manera integrada, en sistema, es posible lograr hacer frente a este reto del entorno con el empleo de este enfoque.

En cualquier tipo de organización, pero fundamentalmente en las prestadoras de servicios la Gestión por Competencias tiene una gran importancia. Sin embargo, la gestión de las competencias requiere de su medición y control.

Por consiguiente, el objetivo general de este estudio es aplicar un procedimiento para la definición de escalas de medición e indicadores de control de la gestión de competencias básicas en procesos clave, que haga posible el logro de la efectividad y eficacia de los mismos. Los objetivos específicos que contribuyen a su logro se refieren a: Construir las bases teórico-referenciales de la investigación; Efectuar un análisis crítico sobre los procedimientos para definir indicadores de gestión en procesos clave; Aplicar un procedimiento para la definición de escalas de medición e indicadores de control de la gestión de competencias básicas de procesos clave en el proceso seleccionado, y Proponer indicadores de efectividad y eficacia para evaluar las propuestas de medición en su aplicación, como resultado de la implantación del procedimiento en períodos posteriores. 
Durante el desarrollo de la investigación se emplean los métodos de análisis y síntesis, de expertos, la dinámica de grupos y de solución de problemas. De igual forma se utilizan instrumentos y técnicas de interrogación (cuestionarios y encuestas) el enfoque sistémico y herramientas estadístico matemáticas para la aplicación de instrumentos de medición, así como para la validación y fiabilidad de los resultados tales como el Análisis Multivariante.

\section{METODOLOGÍA:}

La propuesta metodológica de la investigación desarrollada, está compuesta por el modelo teórico seleccionado para dicho propósito, así como el sistema de procedimientos para el diseño de indicadores de gestión de competencias distintivas o básicas de procesos claves, bajo una concepción sistémica. En el presente trabajo, se hace referencia a dicho modelo, se presenta el procedimiento, así como los principales resultados de la aplicación de este último. El modelo y el procedimiento general se muestran a continuación, en las Figuras 1 y 2, respectivamente.

Figura 1 Modelo conceptual del sistema de procedimientos para el diseño de indicadores de gestión de competencias básicas de procesos clave

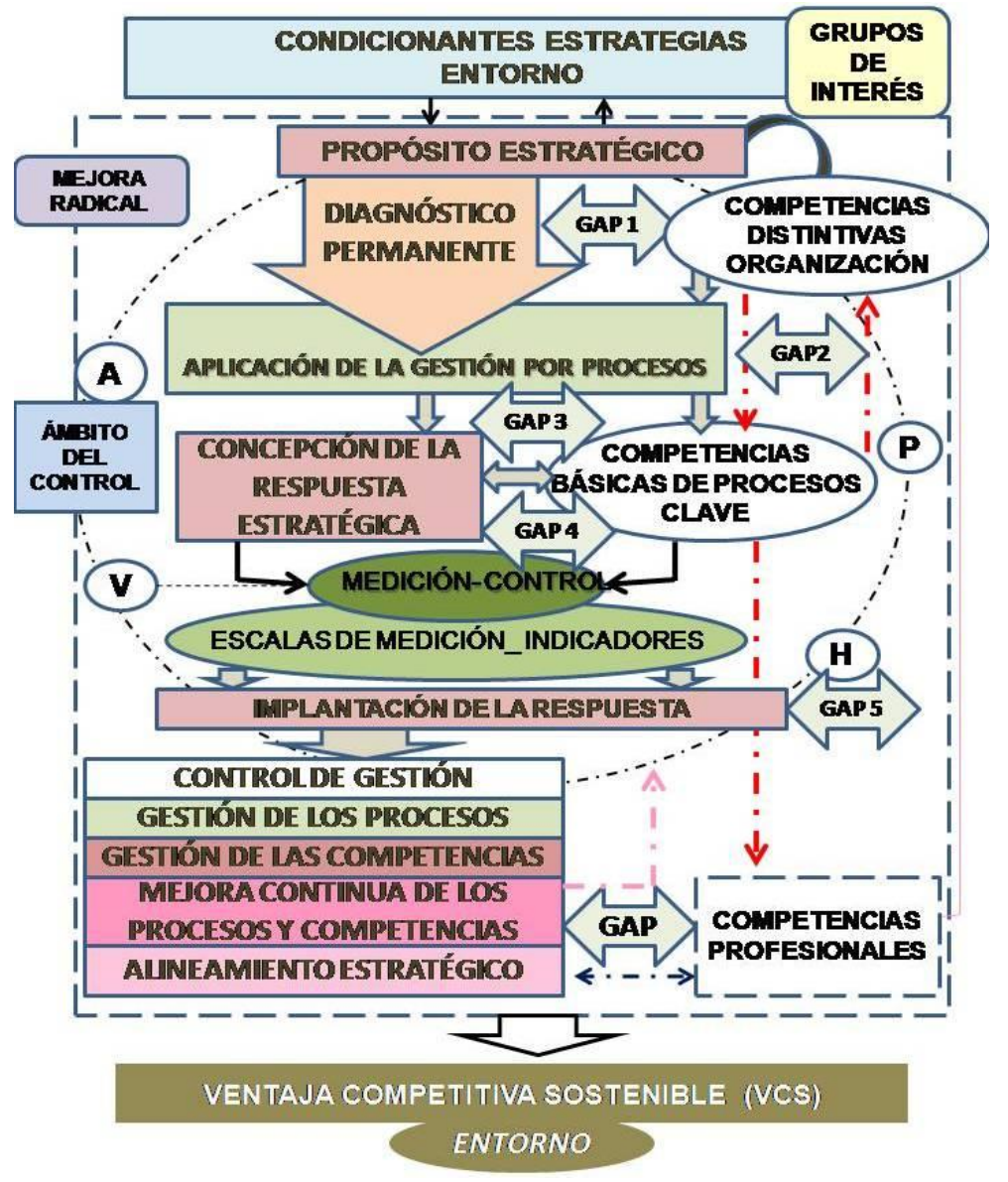

Fuente: Elaboraciòn propia. 
El modelo fue concebido a partir de los aportes realizado por autores, que no solo han abordado en su quehacer la temática del EBC o de la Gestión por Competencias, sino que también han investigado en el campo de la GRRHH en general, del C.G. y del enfoque estratégico (Cuesta, 2005; López, 2008; Hernández, 2008). Tanto el modelo, como el procedimiento, requieren para su implementación, de la práctica de enfoques como el de Gestión por Procesos, el estratégico, la gestión de la Cultura Organizacional (C.O), así como del Control de Gestión moderno.

Figura 2 Procedimiento general para el diseño de indicadores de gestión de competencias básicas de procesos clave.

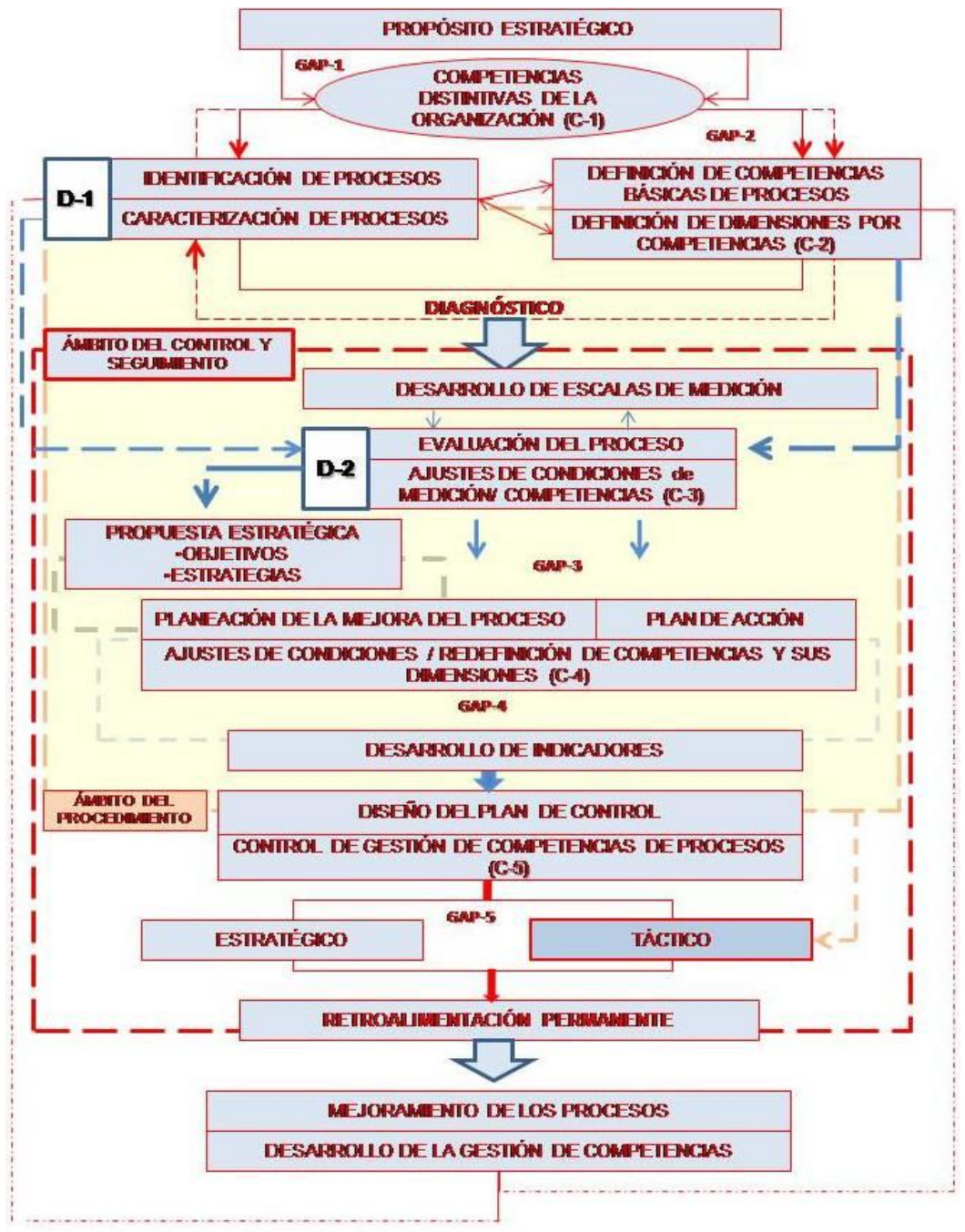

Fuente: Elaboraciòn propia.

El procedimiento general para el diseño de indicadores de control de la gestión de competencias básicas de procesos clave, se estructuró en cuatro (4) fases, de los dos momentos generales del ciclo gerencial: Planeación e Implantación. No obstante, en función del alcance de la investigación, fue desarrollado sólo el primer momento: el diseño del sistema. La implantación, (generalización) corresponde a investigaciones que continúan actualmente. En un primer momento del procedimiento 
(Planeación o diseño del sistema), fueron concebidas las 4 fases siguientes: Identificación, caracterización y evaluación de los procesos; Definición de las competencias básicas del proceso clave; Construcción de las escalas de medición; Definición de los indicadores de gestión de las competencias, desarrolladas a través de los procedimientos de apoyo diseñados al afecto.

Todos los procedimientos cumplieron una función específica para contribuir a garantizar la concepción en sistema de la medición y control de las competencias en los procesos. Con este fin, en la Figura 3, se representan las interrelaciones entre los procedimientos mencionados a partir de la función de cada uno de ellos en el sistema.

Figura 3 Sistema de procedimientos para la definición de indicadores de gestión de competencias básicas de procesos clave

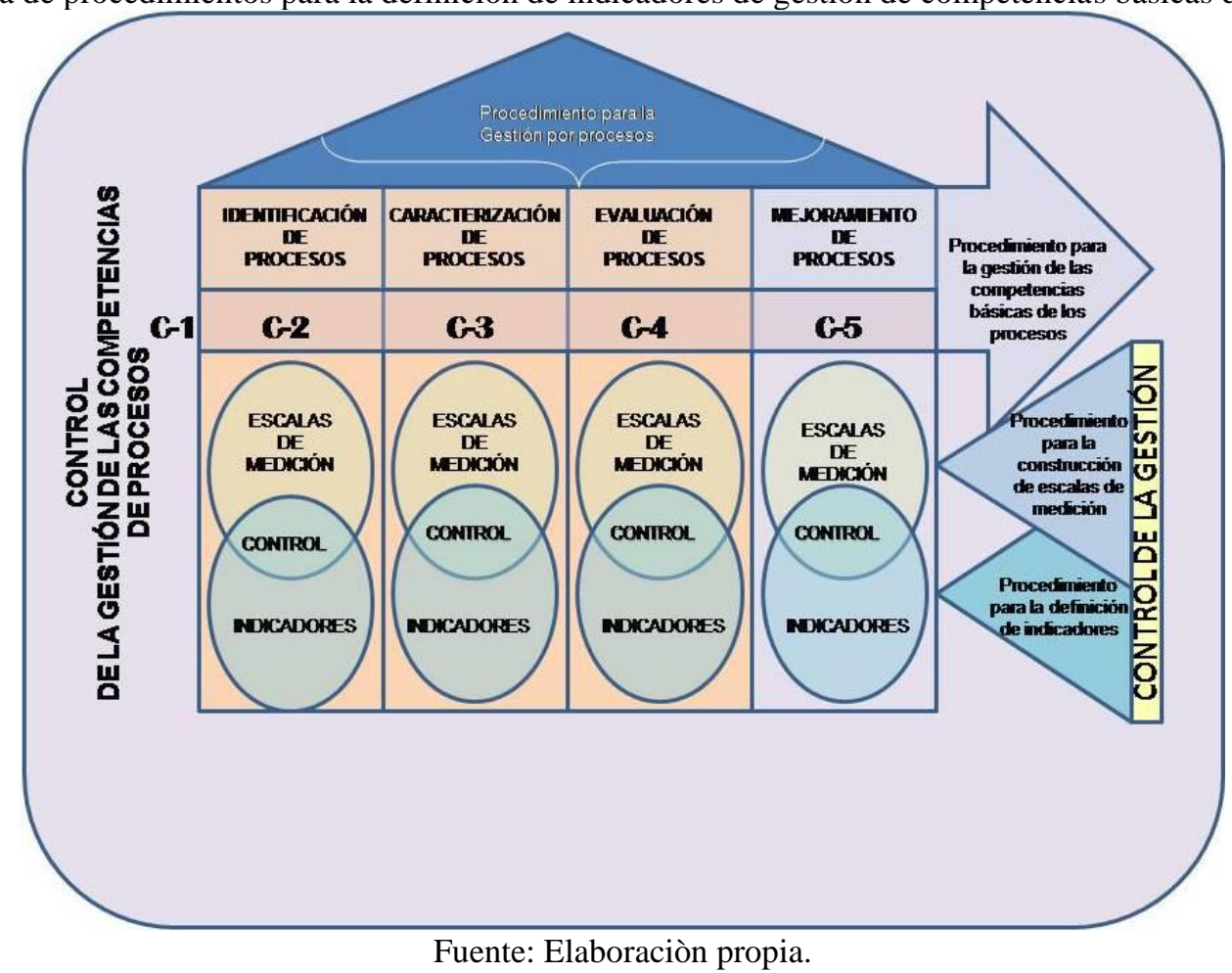

El procedimiento 1, para la gestión por procesos (Villa, 2006) tomó como fundamento el mecanismo de actuación del ciclo Gerencial Básico de Deming (P-HV-A), e incorpora herramientas de enfoques avanzados de la gestión de la calidad, tales como la metodología Seis Sigma.

El procedimiento 2, para la determinación de competencias básicas de procesos clave, (Hernández, 2008), completó las bases sobre las que se sustenta el sistema de procedimientos y constituye la vía que contextualiza la definición de indicadores de gestión, en este caso dirigido también a la mejora de los procesos en su contribución al alineamiento estratégico, al tratarse de la gestión de competencias, mediante la cual se crean las directrices del trabajo con las competencias profesionales o del puesto. 
El procedimiento 3 para el desarrollo de escalas de medición de la gestión de competencias en los procesos, aportó la precisión necesaria para la medición de un activo tan intangible de las organizaciones como son sus competencias. Esta vía, que constituye el empleo de escalas de medición favorece la manipulación efectiva de variables y dimensiones que conforman las competencias y su gestión, para la posterior definición de indicadores con un elevado nivel de ajuste a las condiciones concretas de medición y control de las mismas.

No se trata, por tanto, de ejercer el control como un fin en sí mismo, sino como un medio para lograr gestionar competencias y que ese medio posea las características de flexibilidad, integralidad y eficacia que exige el control de gestión moderno. Por consiguiente, este procedimiento crea las condiciones de especificidad y contextualización para la medición, requeridas por los criterios de efectividad y eficacia del sistema de indicadores de gestión a diseñar y desarrollar. En la Tabla 1 se muestra un resumen de sus etapas.

Por último, el procedimiento 4, para la definición de indicadores de gestión de competencias básicas de procesos clave que se diseñó para la investigación, es el resultado de los aportes obtenidos en el estado del arte fundamentalmente de las áreas del saber del control de gestión, gestión por procesos, la gestión de competencias y la mejora continua, así como otros igualmente relevantes para la gestión moderna como lo es el de TQM, en el que ha intervenido la labor continuada de diversos autores (Amat,2000; Juran y Blanton, 2001; Beltrán,2005; Cuesta, 2005). Los elementos que componen el procedimiento, se muestran en la Tabla 2.

Este procedimiento específico que tributa directamente al propósito a lograr en el trabajo y a su aporte metodológico, completa el enfoque de sistema que sirve de base al Procedimiento General y sus instrumentos, métodos y herramientas asociadas a cada una de sus etapas, ya que abarca todos los momentos que la integración de enfoques en función de la gestión integral en mejora continua de las competencias en la organización requiere. 
Tabla 1 Elementos componentes del procedimiento para el diseño de escalas de medición de competencias básicas de procesos.

\begin{tabular}{|c|c|c|c|}
\hline ETAPA & OBJETIVOS & $\begin{array}{l}\text { CRITERIOS } \\
\text { PARAEVALUAR }\end{array}$ & $\begin{array}{l}\text { MÉTODOS Y HERRAMIENTAS } \\
\text { (más significativos) }\end{array}$ \\
\hline $\begin{array}{l}\text { 1.-DEFINICIÓN } \\
\text { DEVARIABLES }\end{array}$ & $\begin{array}{l}\text { Definir los elementos } \\
\text { medbles decada } \\
\text { competencia } \\
\text { (Conceptualizar, } \\
\text { operacionalizary } \\
\text { definir indicadores } \\
\text { para cada variable) }\end{array}$ & $\begin{array}{l}\text {-Adecuación al } \\
\text { contexto } \\
\text {-Confiabilidad } \\
\text {-Validez }\end{array}$ & $\begin{array}{l}\text { Trabajo de grupo } \\
\text { Grupo de expertos } \\
\text { Confiabilidad portest-retest } \\
\text { Formas alternativas o paralelas } \\
\text { Mitades partidas } \\
\text { CoeficienteAlphade Cronbach } \\
\text { CoeficienteKR-20 Kuder \& Richardson }\end{array}$ \\
\hline $\begin{array}{l}\text { 2.-DEFINICIÓN } \\
\text { DEATRIBUTOS } \\
\text { OBSERVABLES }\end{array}$ & $\begin{array}{l}\text { Definir los } \\
\text { requerimientos } \\
\text { medbles dela gestión } \\
\text { de las competencias } \\
\text { (Definiciónde ítems) }\end{array}$ & $\begin{array}{l}\text {-Integraciónal } \\
\text { contexto } \\
\text { estratégico } \\
\text {-Beneficio a la } \\
\text { mejora delproceso } \\
\text {-Factibilidad de } \\
\text { medición y gestión }\end{array}$ & $\begin{array}{l}\text { Trabajo de grupo } \\
\text { Grupo de expertos } \\
\text { Análisis factorial de componentes } \\
\text { principales } \\
\text { Análisis factorial confirmatorio(LISREL) }\end{array}$ \\
\hline $\begin{array}{l}\text { 3.-DISEÑO DEL } \\
\text { INSTRUMENTO } \\
\text { DE MEDICIÓN }\end{array}$ & $\begin{array}{l}\text { Diseñar los } \\
\text { instrumentos de } \\
\text { medición que faciliten } \\
\text { la contextualización de } \\
\text { cada indicador }\end{array}$ & $\begin{array}{l}\text {-Validezde } \\
\text { constructo } \\
\text {-Validezde } \\
\text { contenido } \\
\text {-Validezde criterio } \\
\text {-Fiabilidad }\end{array}$ & $\begin{array}{l}\text { Grupo de expertos } \\
\text { Prueba de Kendall } \\
\text { Análisis factorial } \\
\text { Alpha de Cronbach }\end{array}$ \\
\hline
\end{tabular}

Fuente: Elaboración propia.

Tabla 2 Elementos componentes del procedimiento para el diseño de indicadores de gestión de competencias de procesos clave.

\begin{tabular}{|c|c|c|}
\hline EUAPA & CBJETIVOS & $\begin{array}{c}\text { METOEOS Y HERRAVIIENTAS } \\
\text { (mäs significativos) }\end{array}$ \\
\hline $\begin{array}{l}\text { 1.-DEFINICIÓN DE } \\
\text { LOS } \\
\text { REQUERIMIENTOS } \\
\text { MEDIBLES }\end{array}$ & $\begin{array}{l}\text { Defnir los requerimientos de } \\
\text { medición de la gestión, entre } \\
\text { los límites de sosteníbilidad } \\
\text { y de integración de cada } \\
\text { competencia }\end{array}$ & $\begin{array}{l}\text { Trabajo de grupo } \\
\text { Grupo de expertos } \\
\text { Análisis factorial de componentes principales } \\
\text { Análisis factorial confimatorio (Path Analysis) }\end{array}$ \\
\hline $\begin{array}{l}\text { 2.-DETERMINACIÓN } \\
\text { DELABRECHA }\end{array}$ & $\begin{array}{l}\text { Definir las necesidades de } \\
\text { ajuste por las diferencias } \\
\text { existentes entre los } \\
\text { requerimientos de medición } \\
\text { de la gestión de la } \\
\text { competencia y la situación } \\
\text { actualde las mediciones }\end{array}$ & $\begin{array}{l}\text { Consulta de documentos } \\
\text { Trabajo de grupo } \\
\text { Grupo de expertos } \\
\text { Prueba de Kendall } \\
\text { Instrumentos de interrogación } \\
\text { Alpha de Cronbach } \\
\text { Análisis factorial de componentes principales }\end{array}$ \\
\hline $\begin{array}{l}\text { 3.-DISEÑO } \\
\text { SISTEMA DEL } \\
\text { INDICADORES EN EL } \\
\text { PLANDE CONTROL }\end{array}$ & $\begin{array}{l}\text { Diseñar el plan de control } \\
\text { que integre el sistema de } \\
\text { indicadores, el rango y las } \\
\text { medidas de control a } \\
\text { efectuar para la mejora } \\
\text { continua }\end{array}$ & $\begin{array}{l}\text { Consulta dedocumentos } \\
\text { Grupo de expertos } \\
\text { Trabajo de Grupo } \\
\text { Análisis factorial de componentes principales } \\
\text { Diagramas de control } \\
\text { Hojas de verificación }\end{array}$ \\
\hline
\end{tabular}

Fuente: Elaboración propia.

\section{RESULTADOS}

Para efecto de su validación, el procedimiento fue aplicado al proceso de selección e inducción del talento humano de una organización comercializadora. Con el propósito de definir mediante experiencia piloto, las escalas de medición e indicadores que directamente incidan en la gestión y mejora de las competencias básicas de procesos en correspondencia con las distintivas de la organización. Antes de la presente investigación no se tenían antecedentes de definición de competencias en los niveles organizacional y de procesos ni de su medición y gestión. 
Para la selección de este proceso clave, mediante el Método de Expertos se recurrió al método de los Factores Críticos de Éxito (FCE). La determinación del número de expertos (17) para la investigación, se calculó mediante un modelo binomial. Los requisitos que debía cumplir cada experto para demostrar su competencia a los efectos del presente trabajo, fueron objeto de análisis de una investigación precedente (Bermúdez, 2007).

El procedimiento se validó en el proceso seleccionado durante un período de seis meses posteriores a la propuesta. Por estar fundamentado en un enfoque de mejoramiento continuo, y dirigido al logro de la gestión en sistema de las competencias en los niveles estratégico y táctico de dirección, la efectividad y la eficacia del propio procedimiento general, también fueron medidas y evaluadas, en función de validar, entre otros efectos de importancia para la investigación y la validez de la hipótesis de la misma.

Por ello, dado que el control es una función que se compone en actividades que no añaden valor ni a los procesos ni a los clientes, en el caso de la investigación se propuso el diseño de un grupo de indicadores. Teniendo en cuenta la variedad de constructos existentes al respecto de los criterios de efectividad y eficacia, así como el propósito y alcance de la investigación, se conceptualizaron las mismas para el contexto del trabajo, quedando definidas como: efectividad del control de la gestión competencias básicas de procesos, cuando la gestión de las competencias responde a los requerimientos de integración y sostenibilidad de la competencia en un ciclo de mejora continua y de alineamiento estratégico y eficacia del control de gestión de competencias básicas de procesos; cuando se alcanzan los niveles de integración y de sostenibilidad (contextualización) para un período de planeación dado en un ciclo de mejoramiento definido, dentro del rango de control aprobado, al menor costo posible en cada acción de control.

De esta manera, el proceso de evaluación del control sobre los procedimientos y sus resultados, se hace posible a través de un grupo de indicadores, que en sistema responden a cada una de las dos dimensiones y se muestran en la Tabla 3. 
Tabla 3 Sistema de indicadores para evaluar el sistema de procedimientos

\begin{tabular}{|c|c|c|c|}
\hline DIMENSIÓN & DEFINICIÓN & INDICADORES & EXPRESION \\
\hline \multirow{3}{*}{$\begin{array}{l}\text { Efectividad de } \\
\text { las } \\
\text { mediciones } \\
\text { para el control } \\
\text { de la gestión } \\
\text { de } \\
\text { competencias } \\
\text { básicas de } \\
\text { procesos } \\
\text { ctave }\end{array}$} & \multirow{3}{*}{$\begin{array}{l}\text { Cuando la medición } \\
\text { de la gestión de las } \\
\text { competencias } \\
\text { responde a los } \\
\text { requerimientos de } \\
\text { infegración y } \\
\text { sostenifindad de ta } \\
\text { compefencia en un } \\
\text { ciclo de mejora } \\
\text { continua y de } \\
\text { alinearniento } \\
\text { estratégion. }\end{array}$} & $\begin{array}{l}\text { Îndice de efectividad de la medición } \\
\text { en las escalas (IFE) siendo: } \\
\text { KEIO > 0,5 en cadla escala } \\
\text { PEB < } 1 \% \text { en cada escala } \\
\text { Donde: EF (Escalas efectivas) } \\
\text { IE (Total de escalas) }\end{array}$ & IEE $=E E I T E * 100$ \\
\hline & & $\begin{array}{l}\text { Indice de efectividad de los } \\
\text { indicadores (If) siendo IE los que } \\
\text { responden a mejoras de } \\
\text { competencias (sostenibidad) } \\
\text { Donde: IE (Indicadores efectivos) } \\
\text { II (Total de indicadores) }\end{array}$ & $\mathrm{IE}=\mathrm{IE} / \mathrm{T} * 100$ \\
\hline & & $\begin{array}{l}\text { Îndice de integración de } \\
\text { indicadores (ill) siendo II I os que } \\
\text { se integran al resto de las acciones } \\
\text { de condrol (integración) } \\
\text { Donde: II (indicadores integrados) } \\
\text { TI (Total de indicadores }\end{array}$ & $\mathrm{III}=\mathrm{II} / \mathrm{TI} * 100$ \\
\hline \multirow{2}{*}{$\begin{array}{l}\text { Eficacia de las } \\
\text { mediciones } \\
\text { para el } \\
\text { Control de } \\
\text { gestión de } \\
\text { corroctencias } \\
\text { básicas de } \\
\text { procesos }\end{array}$} & \multirow{2}{*}{$\begin{array}{l}\text { Cuando se } \\
\text { satisfacen con las } \\
\text { mediciones de las } \\
\text { competencias los } \\
\text { requerimientos de } \\
\text { los clientes del } \\
\text { proceso, dentro del } \\
\text { rango de condrol } \\
\text { aprobado, } \\
\text { competencias en } \\
\text { vinciladas a } \\
\text { actividades que } \\
\text { responden a ARC }\end{array}$} & $\begin{array}{l}\text { Índice de eficacia de la medición } \\
\text { en las escalas } \\
\text { (IEaE) siendo: } \\
\text { Fiabilidad > 0,8 en cada escala } \\
\text { Donde: TEaE (Escalas eficaces) } \\
\text { TE (Total de escalas) }\end{array}$ & IEaE=EaE $/$ TE $* 100$ \\
\hline & & $\begin{array}{l}\text { Índice de eficacia de los } \\
\text { indicadores (IEal) siendo IEa I os } \\
\text { que responden a acciones de } \\
\text { condrol que forman parte de } \\
\text { actividades que responden a ARC } \\
\text { Donde: IEa (Indicadores eficaces) } \\
\text { TI (Total de indicadores) }\end{array}$ & IEal =IEa $f \Pi * 100$ \\
\hline
\end{tabular}

Fuente: Elaboración propia.

Todo proceso de transformación, con cuyo fin se aplica cualquier procedimiento, debe entenderse como un cambio. Para que éste tenga éxito el cambio debe tener dos características fundamentales: ha de ser una solución de calidad para el problema del sistema en términos de validez técnica y lógica y, en segundo lugar, debe ser aceptable para los miembros del sistema que tiene el problema, es en este sentido en el que se enfoca la dimensión de efectividad a la que se acudió en la investigación y los dos indicadores que se propusieron para medirla.

De esta forma, una vez definidos las mejoras requeridas por el proceso clave seleccionado y las correspondientes competencias básicas del mismo, se obtuvo que los indicadores del proceso, ya no se correspondían con las necesidades específicas de su gestión. Es por ello que, a partir de la información brindada por el Plan de Mejora del proceso, se propuso por el equipo de trabajo y expertos, un sistema de indicadores para la gestión de las competencias básicas del mismo, definiéndose sus límites de control. A 
modo de ejemplo, de manera sintética, en la Tabla 4, se muestran algunas competencias básicas del proceso clave seleccionado con sus requerimientos de medición e indicadores para medirla.

Tabla 4 Competencias y sus indicadores.

\begin{tabular}{|c|c|c|c|}
\hline COMPETENCIAS & $\begin{array}{c}\text { ATRIBUTOS OBSERVABLESA } \\
\text { MEDIR }\end{array}$ & INDICADORES & $\begin{array}{l}\text { RANGO DE } \\
\text { CONTROL }\end{array}$ \\
\hline $\begin{array}{l}1 \text { Capacidad de mantener la orientación } \\
\text { estratégica y hacia las competencias, en } \\
\text { la gestión en el día a día de la selección } \\
\text { y la inducción }\end{array}$ & $\begin{array}{l}\text { Modo de hacer las cosas. } \\
\text { Funcionamiento del proceso. } \\
\text { Requerimiento de los clientes. } \\
\text { Orientación estratégica. }\end{array}$ & $\begin{array}{l}\text { Nivel de cumplimiento de la } \\
\text { misión del proceso } \\
\text { Nivel de satisfacción de los } \\
\text { clientes del proceso }\end{array}$ & $\begin{array}{l}95-100 \% \\
90 \_100 \%\end{array}$ \\
\hline $\begin{array}{l}2 \text { Capacidad de actualización } \\
\text { permanente del flujo informativo en } \\
\text { función de los requerimientos del control, } \\
\text { para la toma de decisiones en la } \\
\text { seleccióne inducción }\end{array}$ & $\begin{array}{l}\text { Retroalimentación eficaz y eficiente } \\
\text { del proceso. } \\
\text { Obtener información constante y real } \\
\text { del proceso. }\end{array}$ & $\begin{array}{l}\text { Nivel de correspondencia del flujo } \\
\text { informativo a las acciones de } \\
\text { mejora del proceso }\end{array}$ & $95 \_100 \%$ \\
\hline $\begin{array}{l}3 \text { Capacidad de empleo de la } \\
\text { informatización segán requerimientos de } \\
\text { la gestión con enfoque de mejora del } \\
\text { proceso de seleccióne inducción }\end{array}$ & $\begin{array}{l}\text { Incorporación de nuevas tecnologias } \\
\text { y saberes. } \\
\text { Explotar de manera eficientey eficaz } \\
\text { las tecnologias. }\end{array}$ & $\begin{array}{l}\text { Resultados de mejora del } \\
\text { proceso vinculados al uso de las } \\
\text { nuevas tecnologias }\end{array}$ & $90 \_100 \%$ \\
\hline $\begin{array}{l}5 \text { Capacidad de renovación y } \\
\text { actualización permanente del proceso de } \\
\text { selección e inducción. }\end{array}$ & $\begin{array}{l}\text { Capacidad de mejoramiento propio } \\
\text { del proceso. } \\
\text { Capacidad de mantener una } \\
\text { permanente retroalimentación del } \\
\text { proceso. }\end{array}$ & $\begin{array}{l}\text { Soluciones de mejora dentro del } \\
\text { propio proceso } \\
\text { Nivel de correspondencia del } \\
\text { flujo informativo con los } \\
\text { indicadores vigentes }\end{array}$ & $\begin{array}{l}90 \_100 \% \\
100 \%\end{array}$ \\
\hline $\begin{array}{l}6 \text { Capacided de aprendizaje continuo } \\
\text { (conocimiento actualizado y permanente } \\
\text { del proceso de selección y de inducción, } \\
\text { as/ como de métodos/herramientas para } \\
\text { su gestión, además de la experiencia } \\
\text { acumulada). }\end{array}$ & $\begin{array}{l}\text { Reducción del tiempo entre aprender } \\
\text { y hacer. } \\
\text { Capacidad de incorporación } \\
\text { constante de nuevas formas de } \\
\text { hacer las cosas. }\end{array}$ & $\begin{array}{l}\text { inmediatez de las respuestas de } \\
\text { mejora en la seleccióny la } \\
\text { integración } \\
\text { Efectividad de las acciones de } \\
\text { mejora en la seleccióny la } \\
\text { integración }\end{array}$ & $\begin{array}{l}95 \_100 \% \\
100 \%\end{array}$ \\
\hline
\end{tabular}

\section{CONCLUSIONES}

El procedimiento general para el diseño de indicadores de gestión de competencias básicas de procesos clave, dirige su utilidad y validez hacia la efectividad y eficacia de las mediciones de la gestión de las competencias distintivas de los procesos priorizados, a través de procesos de control que reporten como resultado de su aplicación, el mejoramiento permanente de los indicadores de cada una de estas dimensiones en la gestión de competencias a nivel de procesos, que como efecto principal contribuya al alineamiento entre los tres niveles de competencias.

$\mathrm{Su}$ sistema de procedimientos y herramientas de apoyo, constituye un importante recurso teóricopráctico para el diseño de indicadores de gestión de competencias a nivel de procesos, permitiendo la integración de diversos aportes de enfoques modernos de gestión en manos de los directivos a los diferentes niveles de dirección, especialmente del nivel táctico, en un instrumento metodológico único, que facilite el logro de la integración de los niveles estratégico y operativo, a través del táctico, tratamiento éste no encontrado en la literatura precedente consultada a los efectos de esta investigación.

La integración de los enfoques basado en competencias, estratégico, de mejora continua, de control de gestión y de gestión por procesos en todas las fases y etapas del sistema de procedimientos, 
hace posible el mejoramiento permanente no sólo de la gestión de las competencias de manera integrada, sino del propio sistema de procedimientos mencionado, a través del diagnóstico permanente.

El sistema de procedimientos concebido para el diseño de indicadores de gestión de competencias de procesos de las organizaciones, puede ser aplicado por su atributo de flexibilidad, a cualquier tipo de organización, siempre y cuando el punto de partida de su diseño sea la identificación de las particularidades de cada organización, de su estado de desarrollo, en cuanto a la organización de su gestión y de sus características culturales, entre otras condicionantes. 


\section{REFERENCIAS}

Amat, J. (2000) “Control de Gestión: una perspectiva de dirección”. Edit. Gestión 2000. Barcelona, España, p. 58-78.

Beltrán, J. (1999) “Indicadores de gestión: herramientas para lograr la competitividad”. Edit. 3R Editores. Colombia, p. 85-95.

Bermúdez, Y.(2007) "Procedimiento para el diseño de escalas de medición de la gestión de las competencias de procesos clave de la Sucursal CIMEX Cienfuegos". Proyecto de Curso, Universidad de Cienfuegos, Cuba, p. 1-100

Bermúdez; Y. (2008) "Procedimiento para el diseño de escalas de medición e indicadores de gestión de las competencias de un proceso clave de la Sucursal CIMEX Cienfuegos" Tesis de grado, Universidad de Cienfuegos, Cuba. Jul, p. 1-120.

Cardona, R. (2011) "Estrategia basada en los recursos y capacidades. Criterios de evaluación y el proceso de desarrollo" Revista Electrónica Fórum Doctoral. Vol. 4. ISSN:2027- 2146.

Cabrera, M; Diez Somavilla, R; Sancho, A. (2020) "Ejemplo de proyecto docente innovador: caso Comunica 2". South Florida Journal of Development, Miami, v.1, n.4, p. 258-267, oct/ dec 2020, ISSN 2675-5459.

Cuesta; A. (2005). “Gestión de Competencias” In. La Habana: Editorial Académica. La Habana.

Hernández; J. (2008) "Procedimiento para la determinación de las competencias básicas de los procesos claves en la sucursal CIMEX de Cienfuegos" (online). Disponible en: http://www.eumed.net/cursecon/ecolat/cu/2011/jhs.htm

Jackson; S y Slocum; J. (2014) “Administración. Un enfoque basado en competencias” (online). 11 Edición. Hellriegel, Don; ISBN: 0-324-42140-0.

Juran, J.M. \& Blanton, A. (2001). Manual de Calidad Madrid: Mc Graw Hill. Volumen I. Cap. 5, pp. 199225.

López, F. (2008) "Propuesta metodológica para la integración de la Gestión por Competencias a la Estrategia de las Organizaciones" Tesis doctoral, Departamento de Ingeniería Industrial de la Universidad Tecnológica de la Habana, ISPJAE; Ciudad Habana, Cuba, jul.

Pons, R. et. Al, (2009) “Aplicación de un Procedimiento para la Gestión del Proceso de Investigación de un Departamento Docente” Revista Cubana de Educación Superior (Latindex) Vol. 29.No. 1-2/ 2009. ISSN 0257-4314.

Villa, E. (2006) “Procedimiento para el Control de Gestión en Instituciones de Educación Superior” Tesis doctoral, Departamento de Ingeniería Industrial, Universidad Central "Marta Abreu" de Las Villas, Santa Clara, Villa Clara, Cuba, dic. 2006. 